\title{
EXPOSICIÓN A CONTAMINANTES AMBIENTALES Y PATOLOGÍA RESPIRATORIA
}

\author{
EXPOSURE TO ENVIRONMENTAL CONTAMINANTS \\ \& RESPIRATORY PATHOLOGY
}

\author{
Ma EUGENIA GONZÁLEZ DOMÍNGUEZ ${ }^{(1)}, \mathrm{M}^{\mathrm{a}}$ DOLORES RUEDA YGUERAVIDE ${ }^{(2)}$, \\ DIEGO GUTIÉRREZ FERNÁNDEZ ${ }^{(2)}$, JUAN MANUEL GÓMEZ GUTIÉRREZ ${ }^{(3)}$, \\ ANTONIO LEÓN JIMÉNEZ(4), JOSÉ PÉREZ ÁLVAREZ ${ }^{(5)}$.
}

(1) Facultativo especialista en Medicina del Trabajo. Responsable del Departamento de Salud Laboral del Centro Bahía de Cádiz de EADS- CASA. El Puerto de Santa María. Cádiz. España.

(2) Facultativos especialistas de área de Alergología.

Unidad de gestión clínica de Neumología y Alergia. Hospital Puerta del Mar. Cádiz. España.

(3) Facultativo especialista de área de Neumología.

Unidad de gestión clínica de Neumología y Alergia. Hospital Puerta del Mar. Cádiz. España.

(4) Jefe de Sección de Neumología y Alergia.

Unidad de gestión clínica de Neumología y Alergia. Hospital Puerta del Mar. Cádiz. España.

(5) Ingeniero Industrial y Técnico Superior en Higiene Industrial.

Servicio de Prevención Mancomunado de EADS-CASA, AIRBUS y EADS CASA ESPACIO.

\section{RESUMEN}

Objetivo: Tras la aparición de 5 casos con sintomatología respiratoria en una empresa aeronáutica y su exposición a diferentes sustancias químicas, nos propusimos investigar su posible relación con el medio laboral. Material y métodos: Se realizaron mediciones ambientales de polvo de aluminio, tricloroetileno y glicol, estudio alergológico con dichas sustancias además de neumoalergenos y tests epicutáneos con batería estándar europea, análisis de sangre, radiografías de tórax, mediciones de flujo pico $\mathrm{y}$ pruebas funcionales respiratorias, test de metacolina y electrocardiograma. Resultados: La medición del flujo pico y el test de hiperreactividad bronquial directo con metacolina fueron negativos. El estudio alergológico de las sustancias expuestas fue negativo detectándose en 3 casos resultados positivos a neumoalergenos. Conclusiones: La negatividad en las mediciones del flujo pico y el test de metacolina estando el trabajador en activo, prácticamente descartarían un cuadro de asma laboral, en la actualidad. No podemos descartar la posibilidad concomitante de un Síndrome de disfunción reactiva de la vía aérea en un momento previo al estudio. En tal caso, la existencia de una hiperreactividad bronquial inicial podría haber cedido sin llegar a objetivarse en la actualidad con metacolina.

La creación de equipos multidisciplinarios es fundamental para la detección de enfermedades respiratorias de posible origen laboral.

Palabras clave: Alergia, ocupacional, tricloroetileno, aluminio, glicol.

(Med Segur Trab 2008; 54 (213):41-46)

\section{ABSTRACT}

Target: After the appearance of 5 cases with respiratory symptoms in an aeronautical company and their exposal to different chemical substances, we proposed to investigate their possible link with the working environment. Material and methods: Aluminium environmental dust measurements, trichloroethylene and glycol, alergic study with these substances as well as with aero alergens and epicutaneos tests with a european standard accumulator were made, blood analysis, thorax x-rays, peak-flow measurements and functional respiratory tests, Methacholine test and electrocardiogram. Results: The peak-flow measurement and the direct bronchial hyperactivity test using metacholine were negative. The alergic tests performed were generally negative with the exception of 3 positive results concerning aero alergens test. Conclusions: The negative results of the peak-flow measurements and the metacholine test of an active worker, would practically disguard a cadre of work related asthma, at the present time. We cannot discard the concomitant possibility of a reactive disfunction syndrome via the aerial route in a precise moment of time previous to the study. In such case, the existence of an initial bronchial hyperreactivity could have yielded finally not being detectable with metacholine.

The creation of multidisciplinary equipment is fundamental for the detection of respiratory diseases of possible work origin.

Key words: Allergy, occupational, trichloroethylene, aluminium, glycol.

(Med Segur Trab 2008; 54 (213):41-46)

\section{Correspondencia:}

Dra. María Eugenia González Domínguez

Ctra. de El Puerto de Santa María a Sanlúcar Km. 7,5, Apdo.78.

11500 EL PUERTO DE SANTA MARÍA (Cádiz) España.

Teléfono: 956296044

Correo electrónico: Maria.Gonzalez@ casa.eads.net 


\section{INTRODUCCIÓN}

Desde la revolución industrial y la progresiva introducción de nuevos agentes químicos y procesos industriales en el medio laboral, la prevalencia de las enfermedades ocupacionales se ha ido incrementando. Las enfermedades de origen ocupacional o profesionales constituyen un grupo de procesos patológicos cuya principal característica es la relación causal entre el trabajo y la aparición de la enfer$\operatorname{medad}^{(1)}$.

La relación existente entre trabajo y enfermedad se conoce científicamente desde principios del siglo XVIII con los trabajos de Ramazzini ${ }^{(2)}$. Sin embargo, no fue hasta 1878 en Gran Bretaña donde se llevó a cabo las primeras disposiciones legales "Ley de Fábricas Británicas", en la cual se reconoce que el trabajo es causa directa de enfermedades que pueden evitarse y obliga a adoptar medidas de protección para el trabajador ${ }^{(3)}$.

La legislación laboral ha avanzado desde entonces y se ha ido adaptando al considerable progreso experimentado en el ámbito de la medicina y a los avances de estos procesos industriales. En España, fruto de esta evolución y de la necesidad de actualizar el listado de enfermedades profesionales contenida en el Real Decreto1995/1978, de 12 de mayo, surge el Real Decreto 1299/2006, de 10 de noviembre, con el nuevo cuadro de enfermedades profesionales $^{(4)}$. En el 2008, durante los meses de Enero y Febrero, el mayor número de partes comunicados por enfermedad profesional ha sido por agentes físicos $(82,71 \%)$, en segundo lugar por enfermedades de la piel $(6,28 \%)$ y en tercer lugar las enfermedades producidas por agentes químicos $(4,57 \%)^{(5)}$.

Dentro de las enfermedades profesionales, el espectro de la patología respiratoria ocupacional es amplia y variada, ya que los agentes inhalados pueden producir alteraciones de las vías aéreas y/o de las zonas de intercambio gaseoso, gran parte de su conocimiento es resultado de la epidemiología clínica. El estudio de los factores de riesgo, la presencia de enfermedad y la relación exposición-enfermedad ha permitido progresar en la prevención de estas enfermedades ${ }^{(6)}$.

La prevención tiene pues en las enfermedades profesionales un significado especialmente importante, porque de verdad, puede evitar o disminuir la aparición de la enfermedad ${ }^{(7)}$.

\section{OBJETIVOS}

El objetivo de nuestro trabajo es detectar la posible relación entre la sintomatología respiratoria presentada por 5 trabajadores del área de chapistería de una empresa aeronáutica y su exposición a diferentes sustancias en el lugar de trabajo.

\section{MATERIAL Y MÉTODOS}

Se le realiza un seguimiento durante aproximadamente 11 meses a 5 trabajadores que pertenecen al área de chapistería y que presentan diferentes síntomas respiratorios. Las características de los trabajadores son las siguientes: los 6 trabajadores son exfumadores de más de 15 años, todos se encuentran expuestos desde el año 2003 al mismo ambiente laboral. Entre los antecedentes personales destaca rinitis en uno de los casos, sinusitis desde hace más de 20 años con episodios de repetición ocasionales en otro caso y un tercer caso intervenido de sinusitis y tabique nasal presentando además urticaria colinérgica en primavera y otoño con prurito en la ducha. La sintomatología respiratoria que presentan es variable: tos seca al realizar esfuerzos y con la risa, tos y expectoración, sensación de taponamiento nasal, disnea, sibilantes, y dolor en tórax y espalda. (Tabla I) .

El estudio se ha llevado a cabo por un equipo multidisciplinar formado por alergólogos, higienista industrial, médico del trabajo y neumólogos durante un período que incluye los años 2006 y 2007.

Se realizaron mediciones higiénicas de tipo ambiental de las sustancias a las que pueden estar expuestos dichos trabajadores: polvo de aluminio ${ }^{(8)}$ ( 3 trabajadores expuestos), tricloroetileno (6 trabajadores expuestos) y glicoles (6 trabajadores expuestos).

Dentro del muestreo activo del medio ambiente, la captación de los contaminantes químicos por fijación y concentración de los mismos sobre soporte constituye la técnica más ampliamente utilizada. El paso forzado de aire, que contiene sustancias químicas potencialmente peligrosas a través de un soporte, lleva consigo la generación del muestreo de campo.

Para la realización del muestro de los contaminantes químicos se han seguido los siguientes criterios generales:

1. Los contaminantes a muestrear se han seleccionado teniendo en cuenta la composición de los productos utilizados en el proceso generador de la contaminación y la forma en la que se genera, así como la peligrosidad (desde el punto de vista higiénico) y la posible evaluación de cada contaminante.

Por tanto, se opta por muestrear y evaluar aquellos contaminantes de los que se supone pueden estar presentes en el ambiente de trabajo. 
Tabla I: Resultados de las pruebas médicas realizadas y características de los trabajadores

\begin{tabular}{|c|c|c|c|c|c|c|c|}
\hline & $\begin{array}{l}\text { Antecedentes } \\
\text { personales } \\
\text { relacionados }\end{array}$ & Cuadro clínico & $\begin{array}{c}\text { Tiempo con } \\
\text { síntomas }\end{array}$ & $\begin{array}{c}\text { Flujo } \\
\text { pico } \\
\text { (Varia- } \\
\text { bilidad) }\end{array}$ & $\begin{array}{c}\text { Test de } \\
\text { metacolina }\end{array}$ & $\begin{array}{c}\text { Ig E } \\
\text { UI/ } \\
\text { ml }\end{array}$ & $\begin{array}{c}\text { Test cutáneos y } \\
\text { epicutáneos }\end{array}$ \\
\hline $\begin{array}{l}\text { Caso } \\
1\end{array}$ & $\begin{array}{l}\text { No tolera } \\
\text { metamizol } \\
\text { Rinitis }\end{array}$ & $\begin{array}{l}\text { Tos seca } \\
\text { matutina con } \\
\text { esfuerzos y con } \\
\text { la risa, } \\
\text { sibilantes } \\
\text { nocturnos }\end{array}$ & Desde 2003 & $<20 \%$ & Negativo & 76 & $\begin{array}{l}\text { Positivo a } \\
\text { parafenilendiami } \\
\text { na }\end{array}$ \\
\hline $\begin{array}{l}\text { Caso } \\
2\end{array}$ & $\begin{array}{l}\text { Sinusitis desde } \\
\text { los } 20 \text { años con } \\
\text { episodios } \\
\text { ocasionales }\end{array}$ & $\begin{array}{l}\text { Tos y } \\
\text { expectoración }\end{array}$ & $\begin{array}{l}\text { más } \\
\text { agudizado } \\
\text { desde el año } \\
2005\end{array}$ & $<20 \%$ & Negativo & 7,47 & Negativo \\
\hline $\begin{array}{l}\text { Caso } \\
3\end{array}$ & & $\begin{array}{l}\text { Tos crónica } \\
\text { sensación de } \\
\text { taponamiento } \\
\text { nasal } \\
\text { permanenete. } \\
\text { Disnea y } \\
\text { ocasionalmente } \\
\text { opresión } \\
\text { torácica }\end{array}$ & $\begin{array}{l}\text { Desde } \\
\text { finales } 2004\end{array}$ & $<20 \%$ & Negativo & 67 & Negativo \\
\hline $\begin{array}{l}\text { Caso } \\
4\end{array}$ & Control & no & no & $<20 \%$ & Negativo & 8 & Negativo \\
\hline $\begin{array}{l}\text { Caso } \\
5\end{array}$ & $\begin{array}{l}\text { Intervenido de } \\
\text { sinusitis y } \\
\text { tabique nasal, } \\
\text { dermatitis } \\
\text { colinérgica en } \\
\text { primavera y } \\
\text { otoño con } \\
\text { prurito en la } \\
\text { ducha. Posible } \\
\text { intolerancia al } \\
\text { deflazacort. }\end{array}$ & $\begin{array}{l}\text { Sensación de } \\
\text { ahogo }\end{array}$ & Desde 2003 & $<20 \%$ & Negativo & 19 & $\begin{array}{l}\text { Positivos a } \\
\text { Lepidoglyphus } \\
\text { Destructor, } \\
\text { cladosporium, } \\
\text { penicillium }\end{array}$ \\
\hline $\begin{array}{l}\text { Caso } \\
6\end{array}$ & & $\begin{array}{l}\text { Tos seca dolor } \\
\text { de espalda y } \\
\text { tórax sensación } \\
\text { de ahogo }\end{array}$ & Desde 2006 & $<20 \%$ & Negativo & 60 & $\begin{array}{l}\text { Positivo a } \\
\text { derivados } \\
\text { epidermicos de } \\
\text { perro, pólen de } \\
\text { gramíneas, } \\
\text { malezas y olivo }\end{array}$ \\
\hline
\end{tabular}

2 Dadas las características del proceso se han tomado muestras del tipo ambiental, que nos permiten determinar las concentraciones promediadas en el tiempo existentes en un determinado lugar y momento, y que no tienen porque corresponderse con las exposiciones personales, ya que el trabajador, en general, no permanece fijo en el mismo lugar, sino que se desplaza por lugares donde existen diferentes concentraciones de contaminantes en el aire y que además pueden variar en el tiempo.

3. El periodo de muestreo debe ser de suficiente duración y unas condiciones de trabajo habituales para que la muestra pueda considerarse representativa de las verdaderas condiciones y al objeto de reducir al máximo el número de factores esporádicos que pueden dar alteracio- 
nes momentáneas de las concentraciones normalmente existentes.

4. En nuestras determinaciones se han seguido las normas de muestreo contenidas en las hojas de datos del N.I.O.S.H. (National Institute for Occupational Safety and Health) ${ }^{(9)}$ para contaminantes industriales, donde se siguen los criterios aludidos anteriormente.

Los equipos utilizados en la realización del muestreo de contaminantes químicos son los siguientes:

- Bombas de muestreo personal taradas a un caudal aproximado de $21 / \mathrm{mm}$.

- Bombas de muestreo personal taradas a un caudal aproximado de $0,2 \mathrm{l} / \mathrm{mm}$.

- Tubos OVS-2 (XAD-2+FFV $13 \mathrm{~mm}$ ) para determinación de glicoles

- Tubos de carbón activo 50/100 mg para vapores orgánicos.

- Filtros de Acetato de celulosa 0,8 ?m, para determinación de aluminio.

Las determinaciones cualitativas y cuantitativas en laboratorio, adecuadas a cada tipo de muestra, junto a la información disponible sobre la naturaleza de las muestras, nos permiten el cálculo de las concentraciones ambientales existentes durante el tiempo de muestreo. Una vez conocidas las concentraciones ambientales, debemos realizar la valoración del riesgo higiénico asociado a su presencia en el ambiente de trabajo.

Para ello tendremos en cuenta los Limites de Exposición Profesional para Agentes Químicos adoptados por el Instituto Nacional de Seguridad e Higiene en el Trabajo (INSHT) para el año $2006^{(10)}$. Estos límites de Exposición Profesional son valores de referencia para la evaluación y control de los riesgos inherentes a los agentes químicos presentes en los puestos de trabajo (tabla III). Estos valores serán revisados al menos anualmente, en función de las necesidades que plantean los cambios en los procesos de producción y la introducción de nuevas sustancias, de los nuevos conocimientos técnicos y científicos, así como de la evolución del marco legal en el que se apliquen.

Siendo VLA-ED el Valor Límite AmbientalExposición Diaria y es el valor de referencia para la exposición Diaria (ED). Representando condiciones a las cuales se crea, basándose en los conocimientos actuales, que la mayoría de los trabajadores pueden estar expuestos, 8 horas diarias y 40 horas semanales durante toda su vida laboral, sin sufrir efectos adversos para la salud.
Se realizan mediciones de flujo pico mediante medidores portátiles a los 6 trabajadores. Para ello se ha adiestrado previamente al trabajador en el manejo del aparato a la vez que se le especifica por escrito cada uno de los pasos a seguir para realizar las mediciones. Estas mediciones se realizan en 3 ocasiones distribuidas a lo largo del día (previa a la exposición en el lugar de trabajo, durante y una tercera medición fuera de la jornada laboral) durante 15 días, posteriormente se realiza una segunda medición durante 30 días más ${ }^{(11)}$.

El resto de pruebas a realizar son las siguientes: hemograma y velocidad de sedimentación, estudio de coagulación, bioquímica de rutina, inmunoglobulina $\mathrm{E}$ total, radiografía postero-anterior y lateral de tórax, estudio alergológico, test de metacolina, pruebas funcionales respiratorias y electrocardiogra$\mathrm{ma}^{(12)}$.

El estudio alergológico que llevamos a cabo consiste en la realización de tests intraepidérmicos (prick) y tests epicutáneos con las sustancias a las que están expuestos (tricloroetileno, aqua quench (glicol), aluminio en polvo y agua más residuos). Se ha ampliado el estudio testando además neumoalergenos (ácaros, hongos, epitelios, pólenes y látex) y realizando tests epicutáneos con batería estándar europea (True-Test).

\section{RESULTADOS}

En ningún paciente se observó obstrucción bronquial en las espirometrías forzadas realizadas. A pesar de que algunos pacientes refieren clínica respiratoria que pueden relacionar con su lugar de trabajo, no se ha podido objetivar variabilidad en la medición del flujo pico superior al 20\% (medido durante un total de 45 días), ni en el test de hiperreactividad bronquial directo con metacolina. Ambas pruebas diagnósticas tienen una alta sensibilidad y especificidad para el diagnóstico de asma laboral (Tabla I).

Los resultados de los tests intraepidérmicos (prick) y tests epicutáneos realizados con las sustancias a las que estaban expuestos los trabajadores (tricloroetileno, aqua quench (glicol), aluminio en polvo y agua más residuos) fueron negativos en todos los trabajadores, detectándose en 3 casos resultados positivos a neumoalergenos (perro, pólen de gramíneas, malezas, olivo, Lepidoglyphus destructor, cladosporium, penicillium), y un test epicutáneo positivo a parafenilendiamina. La Inmunoglobulina $\mathrm{E}$ total fue normal en todos los pacientes con un valor medio de $39.5 \mathrm{UI} / \mathrm{ml}$.

Los resultados de los muestreos higiénicos se expresan en la tabla II. Por tanto, para los contaminantes con valor VLA-ED establecido, se obtienen 
Tabla II: Resultados de los muestreos ambientales

\begin{tabular}{|c|c|c|c|c|}
\hline $\begin{array}{r}\text { Referencia } \\
\text { muestra }\end{array}$ & $\begin{array}{l}\text { Lugar de } \\
\text { muestreo }\end{array}$ & $\begin{array}{c}\text { Tiempo } \\
\text { muestreo } \\
\text { (minutos) }\end{array}$ & $\begin{array}{c}\text { Contaminante } \\
\text { evaluado }\end{array}$ & $\begin{array}{c}\text { Concentración } \\
\left(\mathbf{m g} / \mathbf{m}^{3}\right)\end{array}$ \\
\hline PVC-1 & $\begin{array}{l}\text { Ambiental sobre } \\
\text { mostrador }\end{array}$ & 60 & Polvo aluminio & 0,04 \\
\hline G-1 & $\begin{array}{l}\text { Ambiental sobre } \\
\text { mostrador }\end{array}$ & 25 & Glicoles & $350-1.000$ \\
\hline G-2 & $\begin{array}{l}\text { Ambiental frente } \\
\text { a pasillo }\end{array}$ & 12 & Glicoles & $350-1.000$. \\
\hline G-3 & $\begin{array}{l}\text { Ambiental sobre } \\
\text { mueble }\end{array}$ & 10 & Glicoles & $350-1.000$ \\
\hline G-4 & $\begin{array}{l}\text { Ambiental frente } \\
\text { a pasillo }\end{array}$ & 10 & Glicoles & $350-1.000$. \\
\hline CA-10 & $\begin{array}{l}\text { Ambiental sobre } \\
\text { mueble }\end{array}$ & 55 & tricloroetileno & 40,00 \\
\hline
\end{tabular}

concentraciones ambientales muy inferiores a estos valores.

Para el caso de los glicoles, indicar que los resultados analíticos de las muestras enviadas al laboratorio recogieron valores inferiores a $2 \mathrm{mg} / \mathrm{tubo}$. Puesto que los límites de detección para glicoles son de $0,7 \mathrm{mg} /$ tubo y que figurarían en los resultados finales como N.D. (no detectado) y los límites de cuantificación están establecidos en $2 \mathrm{mg} /$ tubo, quiere ello decir que los resultados reales del ensayo y por tanto del muestreo están comprendidos entre ambos valores y que calculadas las correspondientes concentraciones ambientales estarán comprendidas entre $350 \mathrm{mg} / \mathrm{m}^{3}$ y $1.000 \mathrm{mg} / \mathrm{m}^{3}$.

Tabla III: Limites de exposición profesional para los contaminantes evaluados

\begin{tabular}{|l|c|c|}
\hline \multirow{2}{*}{ Contaminante } & \multicolumn{2}{c|}{ Limites adoptados } \\
\cline { 2 - 3 } & VLA-ED $\left(\mathbf{m g} / \mathbf{m}^{\mathbf{3}}\right)^{*}$ & Notas \\
\hline Aluminio, metal en polvo & 10 & $\mathrm{C} 2^{* *}$ \\
\hline Tricloroetileno & 273 & No establecido \\
\hline Glicoles & & \\
\hline
\end{tabular}

*VLA-ED: Valor Límite Ambiental-Exposición Diaria

${ }^{* *} \mathrm{C} 2$ : Sustancia carcinogénica de segunda categoría. "Sustancias que, puedan considerarse como carcinogénicas para el hombre. Se dispone de suficientes elementos para suponer que la exposición del hombre a tales sustancias puede producir cáncer. 
Estos compuestos no tienen establecidos valores límite ambiental de exposición, únicamente podemos deducir su presencia en el ambiente durante las operaciones de secado de las piezas después del enjuague en el baño de glicol por la utilización de una muestra de producto como patrón de referencia.

\section{CONCLUSIONES}

1.-Todas las concentraciones ambientales detectadas en el muestreo son inferiores comparadas con su VLA-ED. Los valores para los glicoles no pueden compararse con un VLA-ED, únicamente se detecta su presencia ambiental.

2.-Los resultados del estudio alergológico realizado a todos los trabajadores con las sustancias de exposición en su lugar de trabajo fueron negativos, sin embargo, 2 trabajadores resultaron positivos a diferentes neumoalergenos y 1 trabajador a parafenilendiamina.

3.-A pesar de que algunos pacientes refieren clínica respiratoria que puedan relacionar con su lugar de trabajo, no se ha podido objetivar variabilidad del flujo pico superior al $20 \%$ y el test de hiperreactividad bronquial directo con metacolina fue negativo. Ambas pruebas diagnósticas tienen una alta sensibilidad y especificidad para el diagnóstico de asma laboral, por lo que siendo negativas estando el trabajador en activo, prácticamente descartarían un cua-

\section{$\overline{\text { BIBLIOGRAFÍA }}$}

1. Martínez González C, Rego Fernández G. Enfermedades respiratorias de origen.ocupacional. Arch Bronconeumol 2000; 36(11): 631-644.

2. Ramazzini B: De morbis artificum diatriba. Publicado de origen en Génova en 1719. Traducido por W.C. Wright. University of Chicago Press. Chicago 1940.

3. Sastre J, Quirce S. Patología respiratoria alérgica ocupacional. Emisa; 2003: 35. ISBN: 84-86917-58-1.

4. Asociación de Mutuas de Accidentes de Trabajo (AMAT). Nuevo cuadro de enfermedades profesionales. www.amat.es año 2007. www.enfermedadesprofesionales.net año 2007

5. Ministerio de Trabajo e Inmigración. Seguridad Social. Estadísticas. http://www.seg-social.es/ Internet_1/Lanzadera/index.htm?URL=82 año 2008

6. Meredith S. Reported incidente of occupational asthma in the United Kingdom, 1989-90. J Epidemiol Community Health. 1993; 47(6): 459-63. dro de asma laboral, en la actualidad. Si bien, aunque es poco probable, podrían tratarse de falsos negativos, en cuyo caso la prueba diagnóstica definitiva sería la realización de un test de provocación bronquial específica, con las sustancias de exposición en el lugar de trabajo.

4.- No podemos descartar la posibilidad concomitante de un Síndrome de disfunción reactiva de la vía aérea en un momento previo al estudio, ni de mecanismos no inmunoalérgicos. En tal caso, la existencia de una hiperreactividad bronquial inicial podría haber ido cediendo con el paso del tiempo, sin llegar a objetivarse en la actualidad con el test de metacolina.

La creación de unidades de neumología ocupacional multidisciplinarias constituidas por neumólogos, alergólogos, epidemiólogos, médicos del trabajo, higienistas, etc.. es una valiosa herramienta de trabajo para seguir avanzando en la identificación de las enfermedades de posible origen ocupacional emergentes.

\section{AGRADECIMIENTOS}

la Unidad de gestión clínica de Neumología y Alergia. Hospital Puerta del Mar. Cádiz) así como a Wenceslao Escamilla Tera (Responsable de Seguridad Laboral del Servicio de Prevención del Centro Bahía de Cádiz. El Puerto de Santa María).

7. Hinojosa M.: La prevención en el asma ocupacional..Rev. Esp. Alergol Inmunol Clin 1997; 12: 211-217.

8. O' Donnell TV. Asthma and respiratory problems: a review. Sci Total Environ 1995; 163 (1-3): 137-45.

9. National Institute for Occupational Safety and Health. http://www.cdc.gov/niosh/ año 2006

10. Ministerio de Trabajo y Asuntos Sociales. Instituto Nacional de Seguridad e Higiene en el Trabajo. http://www.mtas.es/insht/practice/vlas.htm año 2006.

11. Miquel-Gomara Perelló J, Román Rodríguez M. Peak-flow meter: technique and utilities in Primary Health Care. Medifam. [serial on the Internet]. 2002 Mar [cited 2008 Mar 31] ; 12 (3): 76-91.

12. Cartier A. Definition and diagnosis of occupational asthma. Eur Respir J 1994; 7(1): 153-60. 